\title{
OSTEOPETRORICKETS IN AN INFANT WITH COEXISTENT CONGENITAL CYTOMEGALOVIRUS INFECTION
}

\author{
Katsafiloudi M*, Gombakis N, Hatzipantelis E, Tragiannidis A \\ *Corresponding Author: Athanasios Tragiannidis, M.D., Ph.D., Assistant Professor of Pediatrics, Pe- \\ diatric Hematology-Oncology Unit, 2nd Pediatric Department, AHEPA Hospital, Aristotle University of \\ Thessaloniki, S. Kiriakidi 1 str., Thessaloniki, Greece. Tel: +306-944-944-777. Fax: +302-310-994-803. \\ E-mail: atragian@ auth.gr; atragian@hotmail.com
}

\begin{abstract}
Osteopetrosis refers to a group of rare hereditary disorders characterized by generalized skeletal densification due to limited bone resorption by osteoclasts. The infantile autosomal recessive form represents the most malignant one with onset early in infancy and life expectancy less than 1-2 years without therapy. Frequently, osteopetrosis is complicated by rickets, a condition called osteopetrorickets. Currently, bone marrow transplantation remains the only treatment option. We present a case of infantile autosomal recessive osteopetrosis complicated by rickets in a 2 and a half-month-old female infant with coexistent congenital cytomegalovirus (CMV) infection, successfully treated by hematopoietic stem cell transplantation (HSCT). Diagnostic procedure and differential diagnosis are discussed along with a short review of the literature. Diagnosis of osteopetrosis requires high clinical suspicion, which is enhanced by radiology and confirmed by bone biopsy and molecular analysis. Our patient has been successfully treated by HSCT and has remained in a good general condition thereafter.
\end{abstract}

Keywords: Congenital cytomegalovirus (CMV); Hematopoietic stem cell transplantation (HSCT); Osteopetrorickets; Osteopetrosis.

\section{INTRODUCTION}

Osteopetrosis, also called "marble bone disease," refers to a group of rare hereditary disorders characterized by osteoclast dysfunction resulting in abnormally dense bone and excessive skeletal mass with paradoxical bone fragility. Heinrich Albers-Schönberg, a German gynecologist and

Hematology-Oncology Unit, $2^{\text {nd }}$ Pediatric Department, AHEPA Hospital, Aristotle University of Thessaloniki, Thessaloniki, Greece radiologist (born in 1891), first described osteopetrosis in 1904 [1]. Since then many types of the disease have been identified, and in 1963, Harry Mullins Worth, a British radiologist (born in 1897) who graduated with degrees in dentistry and medicine, and worked in the UK as well as Canada, introduced the term osteopetrosis (from the Greek "osteo" meaning bone and "petros" meaning stone) because of the rock-like appearance of the bone [2,3]. Osteopetrosis has for decades been categorized by its clinical severity and inheritance pattern into a malignant infantile autosomal recessive form, an intermediate autosomal recessive form and an adult autosomal dominant form, which is the most benign type, frequently identified incidentally $[4,5]$. The incidence of these conditions is estimated about 1 in 250,000 live births for autosomal recessive osteopetrosis (ARO) and about 1 in 20,000 live births for autosomal dominant adult type (ADO) [3]. The disease is more frequently seen in ethnic groups where consanguinity is common [6]. Recent advances in genetics progressively allow the classification of osteopetrosis by its underlying molecular pathogenesis [5]. Mutations in at least 10 genes have been identified in humans, accounting for $70.0 \%$ of all cases, among them TCIRG1, CLCN7 and CAII [3].

Infantile osteopetrosis can manifest with severe bone marrow failure that can mimic hematological malignancy including anemia, thrombocytopenia, leukopenia, susceptibility to infections and hepatosplenomegaly. Narrowing of osseous foramina can lead to compressive cranial neuropathies, vision impairment and deafness [5,7-9]. If therapy is unsuccessful, death occurs by early childhood as a result of bleeding, anemia or infection [9]. In a setting of intense positive body calcium, rickets is a paradoxical complication of osteopetrosis, a condition called osteopetrorickets. This case reports osteopetrorickets in an infant with coexisting congenital cytomegalovirus (CMV), infection, successfully treated by bone marrow transplantation (BMT). 


\section{CASE PRESENTATION}

A full-term 2 and a half-month-old female, the second child of consanguine parents with an uneventful perinatal and neonatal history, was admitted to our clinic due to hepatosplenomegaly, pallor and petechiae noted by their pediatrician at routine check. Upon admission, the infant was afebrile, in a good general condition. Physical examination showed mild growth retardation, hepatosplenomegaly, slightly enlarged anterior fontanelle, abnormal eye movements, mild hypertonia of low extremities. Sporadic petechiae in head, neck and lower abdomen were also noticed. Laboratory findings demonstrated leukocytosis [white blood cell $(\mathrm{WBC}): 41,350 \times 10^{9} / \mathrm{L}$ ], normocytic normochromic anemia [hemoglobin $(\mathrm{Hb}) 7.5 \mathrm{~g} / \mathrm{dL}$, packed cell volume (PCV) $0.24 \mathrm{~L} / \mathrm{L}$, mean corpusucular volume (MCV) $86.9 \mathrm{fL}$, mean corpuscular $\mathrm{Hb}(\mathrm{MCH}) 28.4$ pg, reticulocytes $11.37 \%$ ] and thrombocytopenia [platelet (PLT) count $\left.46,000 \times 10^{9} / \mathrm{L}\right]$. Coagulation exams, direct and indirect Coombs tests were negative. Serology revealed high levels of alkaline phosphatase (ALP) $1290.0 \mathrm{U} / \mathrm{L}$, lactate dehydrogenase (LDH) 1003.0 U/L, lactic acid (18.0 $\mathrm{mmol} / \mathrm{L}$ ) and ferritin $(241.0 \mathrm{ng} / \mathrm{mL})$. Serum calcium (Ca) was in lower limits at $8.2 \mathrm{mg} / \mathrm{dL}$ and phosphorus $(\mathrm{P})$ under normal range $2.3 \mathrm{mg} / \mathrm{dL}$, with a $\mathrm{Ca} \times \mathrm{P}$ product of less than 20. Vitamin $25-\mathrm{OH}-\mathrm{D}$ level was also low $(21.3 \mathrm{ng} / \mathrm{mL})$ and parathyroid hormone was elevated $(17.7 \mathrm{pmol} / \mathrm{L})$. Due to high levels of thyroid-stimulating hormone (TSH) (14.10 $\mu \mathrm{IU} / \mathrm{mL}$ ) indicative of hypothyroidism, the patient was put on levothyroxine therapy. Blood and urine cultures were sterile, stool culture showed normal flora. Infection control was negative for hepatitis B virus (HBV), hepatitis $\mathrm{C}$ virus (HCV), hepatitis A virus (HAV), syphilis, rubella, Ebstein Barr virus (EBV) and toxoplasma.

The CMV DNA was detected in urine and blood and ganciclovir was administered for 21 days. Metabolic control with measurement of organic acids in urine and amino acids in blood and urine was also negative. Abdominal ultrasound evidenced hepatosplenomegaly, heart and brain ultrasonography were normal. Small, grayish papillae and bilateral optic hypoplasia were noted in fundoscopy. Visual and auditory evoked potentials showed absence of waveform (blindness) and conductive hearing loss, respectively. Due to persistent anemia, thrombocytopenia and leukocytosis, a bone marrow aspiration was performed, which excluded leukemia and revealed T and NK cells immunophenotypic disorders. The karyotype was normal. Radiological findings [osteosclerotic elements in X-rays with "bone within bone" appearance (Figure 1), marked sclerosis within the skull base (Figure 2), narrowing of cranial foramina in brain computed tomography (CT) scan] raised the suspicion of osteopetrosis, which was finally

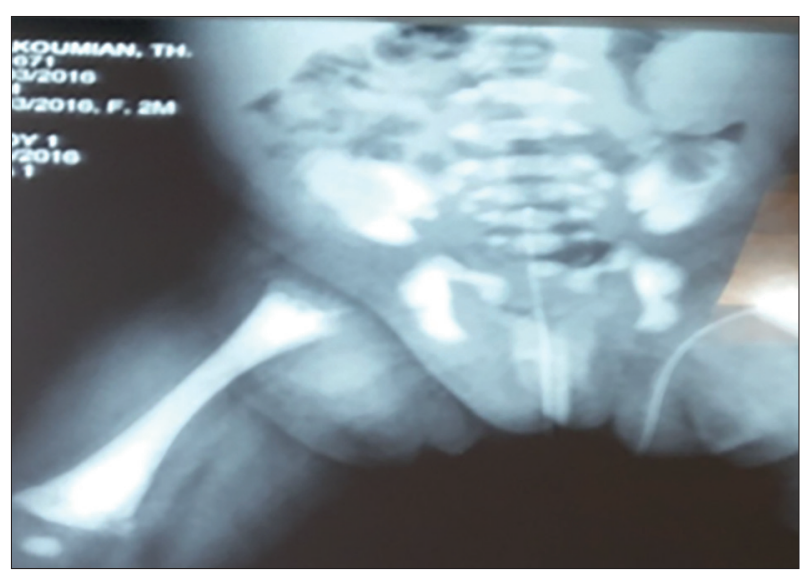

Figure 1. Pelvis-hip-femur X-ray: osteosclerotic.

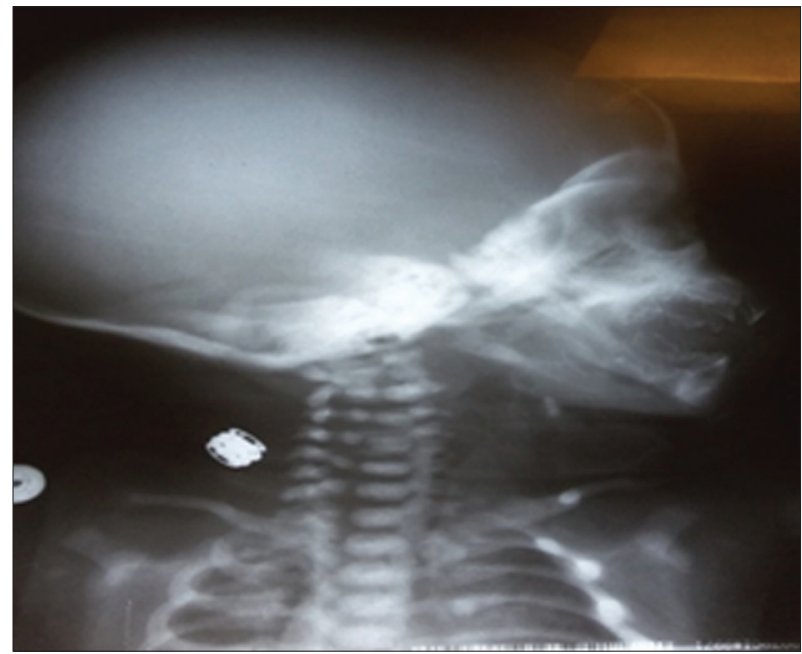

Figure 2. Skull X-ray: sclerotic appearance of "bone within bone" appearance. Cranial base structures.

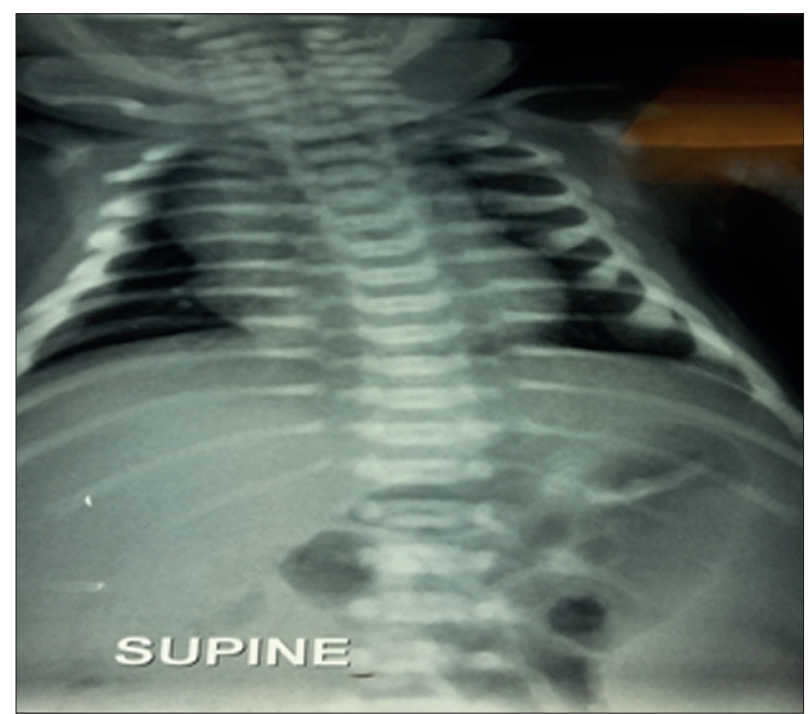

Figure 3. Chest X-ray: rachitic rosary. 
confirmed by bone biopsy and molecular diagnostics. The latter revealed a TCIRG1 gene mutation in a homozygous state, while both parents were found to be heterozygotes for the same allele. Pathology findings were consistent with diagnosis of osteopetrosis. Prominence of the costochondral junctions (rachitic rosary) in chest radiograms (Figure 3), along with low calcium and phosphate levels in serum indicated superimposed rickets.

During hospitalization, the infant remained in good clinical condition and was treated with ganciclovir, ceftazidime due to $E$. coli urinary infection, vitamin $\mathrm{D}_{3}$, levothyroxine and red blood cell (RBC) transfusions. Three months after initial admission, the infant successfully underwent BMT with father being the HLA-compatible donor. Two years later, she remains alive, in a good general condition.

\section{DISCUSSION}

Osteopetrosis seems to be related to defects in the acidification process of resorption lacuna, mainly caused by mutations in the TCIRG1,CLCN7 and CAII genes coding for a 3-subunit of proton pump, chloride channel and carbonic anydrase II, respectively. Mutation in the TCIRG1 or CLCN7 gene is found in nearly $70.0 \%$ of all patients with autosomal recessive osteopetrosis [4,8]. The TCIRG1 gene mutation was also detected in our patient. Mutations in the TCIRG1 gene present clinically as malignant autosomal recessive osteopetrosis in early infancy. Severe anemia, thrombocytopenia, neutropenia, hepatosplenomegaly, susceptibility to fractures, cranial nerve anomalies, visual impairment and deafness, are the prominent manifestations. Meanwhile, CMV infection is the most frequent congenital infection in humans and shares common clinical features including pancytopenia, hepatosplenomegaly, intrauterine growth retardation, sensorineural hearing loss and intracranial calcifications. Our patient was assessed for common causes of pancytopenia and hepatosplenomegaly including CMV and other congenital and acquired infections. Infantile leukemia and myeloproliferating diseases were excluded by bone marrow aspiration. Inborn errors of metabolism and chromosomal anomalies were ruled out by metabolic control and karyotype, respectively. Evaluation for congenital infections revealed a CMV infection, which was regarded as a coincidence, as radiological findings raised the suspicion of osteopetrosis. Other inherited and acquired conditions with sclerotic skeletal appearance such as pyknodysostosis, progressive diaphyseal dysplasia, renal osteodystrophy, hypervitaminosis A and D, chemical poisoning and transient hypersclerosis of infancy were excluded by bone biopsy and genetic analysis that confirmed the diagnosis of autosomal recessive osteopetrosis $[5,10]$.
Remarkable in this case, was the biochemical and radiological evidence of rickets, which is a paradoxical complication of osteopetrosis. Despite a positive total body calcium balance, patients with osteopetrosis tend to develop rickets because the dysfunctional osteoclasts are unable to maintain a normal calcium-phosphorus balance in the extracellular fluid [9]. In osteopetrorickets the serum calcium-phosphate product decreases $(<30.0$ $\mathrm{mg}^{2} / \mathrm{dL}^{2}$ ) so that the mineralization of growing bones is insufficient [4]. The diagnostic radiological findings of rickets superimposed on the osteopetrosis are changes in metaphyses of long bones and in costochondral junctions (rachitic rosary), which was also noticed in our patient, along with the biochemical markers of rickets [4]. Treatment of rickets with calcitriol and calcium supplementation improves the overall condition of these patients and is essential for successful BMT.

At present, hematopoietic stem cell transplantation offers the only chance of cure for malignant infantile osteopetrosis (MIOP), and ideally, it should be performed early, before the irreversible neurologic impairment [7]. Hematopoietic stem cell transplantation (HSCT) using HLA identical donors results in 73.0\% 5-year disease-free survival [3]. The purpose of transplantation is to provide hematopoietic stem cells from which normal osteoclasts can differentiate. However, BMT cannot cure patients with osteopetrorickets, as normal osteoclasts cannot resorb the hypomineralized osteoid. Therefore, it is important to diagnose and treat the rickets before BMT [4].

Another therapeutic option is interferon $\gamma-1 b$, mainly recommended in non infantile osteopetrosis or as a bridge to transplantation [3,5]. Hatzipantelis et al. [11] have reported a case of malignant infantile osteopetrosis treated with IFN- $\gamma$ with subsequent improvement in bone resorption and hematopoietic function but the patient died from sepsis 4 months later. Finally, the therapeutic spectrum includes supportive blood transfusions, treatment of infections and regular assessment of the patient by a multidisciplinary team consisting of specialists in hematology, endocrinology, ophthalmology, neurology-neurosurgery, orthopedics, dentistry, otolaryngology and nephrology. Gene therapy would be the optimum future direction to a radical cure of osteopetrosis. Given the fact that parents of MIOP patients are at $25.0 \%$ risk of having further affected children in each pregnancy, genetic counseling is of utmost importance for the families in whom a mutation has been identified.

\section{CONCLUSIONS}

Malignant infantile osteopetrosis is a rare hereditary skeletal disease, in some cases complicated by rickets. As 
high clinical suspicion is required for diagnosis, MIOP should be kept in mind as a rare cause of pancytopenia and hepatosplenomegaly. Some cases of successful bone marrow transplantation in patients with infantile osteopetrosis exist in the literature, and a similar case of MIOP with coexistent CMV infection has been reported by Lee et al. [8] but in their patient no BMT was performed. To the best of our knowledge, this is the first case of infantile osteopetrosis complicated by rickets in an infant with congenital CMV infection that was successfully treated by HSCT.

Declaration of Interest. The authors report no conflicts of interest. The authors alone are responsible for the content and writing of this article.

\section{REFERENCES}

1. Patil MB. Osteopetro-Rickets: A rare paradoxical association in an infant. J Nepal Paediatr Soc. 2012; 32(1): 88-89.

2. Kocher MS, Kasser JR. Osteopetrosis. Am J Orthop. (Belle Mead NJ). 2003; 32(5): 222-228.

3. Stark Z, Savarirayan R. Osteopetrosis review. Orphanet J Rare Dis. 2009; 4: 5.

4. Gonen KA, Yazici Z, Gokalp G, Ucar AK. Infantile osteopetrosis with superimposed rickets. Pediatr Radiol. 2013; 43(2): 189-195.
5. Wu CC, Econs MJ, DiMeglio LA, Insogna KL, Levine MA, Orchard PJ, et al. Diagnosis and management of osteopetrosis: Consensus guidelines from the Osteo-petrosis Working Group. J Clin Endocrinol Metab. 2017; 102(9): 3111-3123.

6. Kalekar T, Sehrawat V. Autosomal recessive infantile osteopetrosis: Case report with radiological review. Int J Res Med Sci. 2017; 5(6): 2797-2800.

7. Essabar L, Meskini T, Ettair S, Erreimi N, Mouane N. Malignant infantile osteopetrosis: Case report with review of literature. Pan Afr Med J. 2014; 17: 63.

8. Lee SH, Shin JH, Choi BM, Kim YK. A case of cytomegalovirus infection in a neonate with osteopetrosis. Pediatr Infect Vaccine. 2016; 23(1): 72-76.

9. Donnelly LF, Johnson JF 3rd, Benzing G. Infantile osteopetrosis complicated by rickets. Am J Roentgenol. 1995; 164(4): 968-970.

10. Tolar J, Teitelbaum SL, Orchard PJ. Osteopetrosis. N Engl J Med. 2004; 351(27): 2839-2849.

11. Hatzipantelis ES, Gombakis N, Doulioglou V, Bantouraki M, Zafiriou DJ, Katzos G. Infantile type malignant osteopetrosis. Arch Hellenic Med. 2000; 17(2): 189-192. 